\title{
Inhibition of Carotenoid Synthesis in a Mutant of Verticillium albo-atrum
}

\author{
By L. R. G. VALADON AND ROSEMARY S. MUMMERY \\ Department of Botany, Royal Holloway College (University of London), \\ Englefield Green, Surrey
}

(Received 23 May 1966)

\section{SUMMARY}

Verticillium albo-atrum mutant m $5 \cdot 6$ which produces phytoene, $\beta$ - and $\gamma$-carotene, neolycopene $\mathbf{A}$, lycopene and neurosporaxanthin was subjected to the effect of the following inhibitors of carotenoid synthesis: diphenylamine, 2-hydroxydiphenyl, $\beta$-ionone, methylheptenone. Certain intermediates in the Porter-Anderson (1962) pathway for carotenoid biosynthesis not previously present were then observed, namely: phytofluene, $\beta$-zeacarotene, $\zeta$-carotene, neurosporene. When the mutant was grown in the presence of diphenylamine, washed free from it and then grown again in $1 \%$ glucose in $\mathbf{M} / \mathbf{1 5} \mathrm{KH}_{2} \mathrm{PO}_{4}$, the more unsaturated polyenes seem to be formed at the expense of the more saturated ones. There was also evidence that $\gamma$-carotene was formed via $\beta$-zeacarotene and the sequence of carotenoid formation seems to agree very closely to that suggested by Porter \& Anderson (1962).

\section{INTRODUCTION}

As a result of a study on various tomato crosses Porter \& Lincoln (1950) proposed a stepwise interconversion of $\mathbf{C}_{40}$ polyenes as a mechanism of carotenoid biosynthesis, at a time when the exact structures of the intermediates were not known. Porter \& Anderson (1962), with more information available, showed that the original scheme was to a large extent substantiated but had to be revised. The proposed pathway (Fig. 1) suggested that phytoene was dehydrogenated to phytofluene, to $\zeta$-carotene, then to neurosporene.

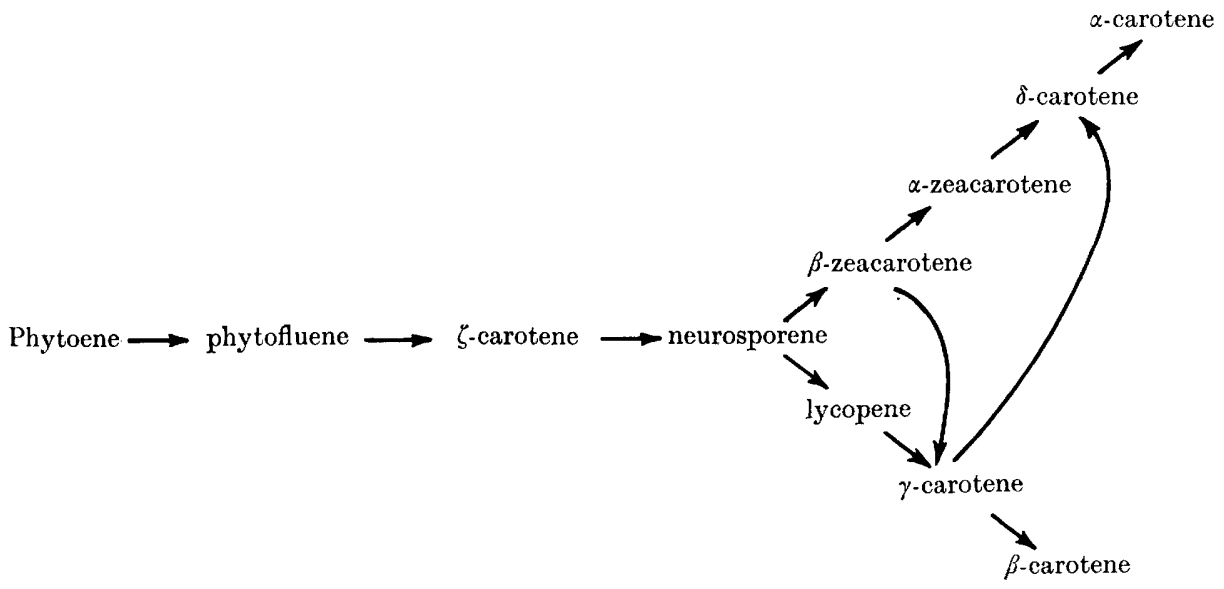

Fig. 1. Pathway of carotenoid biosynthesis suggested by Porter \& Anderson (1962). 
The cyclic $\beta$-carotene could be formed by cyclization of neurosporene to $\beta$-zeacarotene and also by cyclization of lycopene. There seems to be evidence that in fungi, it is neurosporene that is cyclized, and not lycopene: the intermediate $\beta$-zeacarotene has been identified in Phycomyces blakesleeanus (Davies, Villoutreix, Williams \& Goodwin, 1963) and in Rhodotorula glutinis (Simpson, Nakayama \& Chichester, 1964) and it was suggested in both of these that the main pathway between neurosporene and $\gamma$-carotene was mediated through $\beta$-zeacarotene. The structure of $\beta$-zeacarotene as determined by Rüegg et al. may support this view. Davies (1961) who worked on young and older cultures of Rhizophlyctis rosea with $\left[{ }^{14} \mathrm{C}\right]$ labelled mevalonic acid obtained evidence that $\gamma$-carotene was not synthesized from lycopene.

An alternative to the Porter-Lincoln hypothesis was proposed by Goodwin (1952). He suggested that the series of polyenes could be produced independently from a common precursor; this is known as the independent hypothesis. Diphenylamine (DPA) inhibits carotenogenesis and when the colourless cultures of Phycomyces blakesleeanus were resuspended in DPA-free medium, it appeared that $\beta$-carotene was not being formed at the expense of the phytoene series (Goodwin, Jamikorn \& Willmer, 1953). Results obtained by Zalokar (1954) with Neurospora crassa under different conditions of light and oxygen also support this hypothesis, since $\zeta$-carotene, neurosporene and lycopene all appeared simultaneously. Krzeminski \& Quackenbush (1960) by using the incorporation of mevalonic acid-2- ${ }^{14} \mathrm{C}$ into the carotenoids of $N$. crassa showed clearly that the product/precursor relationship between the most unsaturated polyenes and the more saturated ones was not fulfilled. Further, results obtained by Villoutreix (1960) on ultraviolet mutants of Rhodotorula mucilaginosa and also of the effect of 2-hydroxydiphenyl on carotenogenesis support the independent hypothesis. Later work by Goodwin and co-workers (Davies et al. 1963) suggested that $\beta$-zeacarotene, phytofluene, $\zeta$-carotene and neurosporene were formed from phytoene in the presence of DPA, and this is evidence for carotenoid synthesis along the Porter-Anderson (1962) pathway.

Results obtained by Valadon \& Heale (1965) on mutants of Verticillium albo-atrum in which similar amounts of phytoene were obtained in the wild type (which did not contain carotenoids) and in five mutants (which contained carotenoids) did not seem to support the first hypothesis. However, it is known that precursor/product relationships are not always reflected in their quantities, or even in the detectability of the compounds involved (Dr D. G. Anderson, personal communication) and, as Valadon \& Heale (1965) suggested, conclusions based on that type of evidence must be tentative. Inhibitors of carotenoid synthesis are well known, namely diphenylamine (Goodwin, 1952; 'Turian, 1957; Turian \& Haxo, 1954) methylheptenone (Chichester, Wong \& Mackinney, 1954; Nakayama, Chichester, Lukton \& Mackinney, 1957) $\beta$-ionone (Mackinney, Nakayama, Buss \& Chichester 1952); 2-hydroxydiphenyl (Villoutreix, 1960). Usually after treatment with these inhibitors previously unobserved carotenoids are obtained, indicating that pathways for their formation are available in the given organism (Simpson et al. 1964). This prompted further work with $V$. albo-atrum with the hope of elucidating the synthesis of carotenoids in this organism. 


\section{METHODS}

The culture of Verticillium albo-atrum Reinke \& Berthold used in this work is designated $\mathbf{m} 5 \cdot 6$. It is a subculture of the original $\mathbf{5} 5$ orange coloured mutant obtained by Valadon \& Heale, 1965. After about 6 weeks incubation on $2 \%$ malt agar м5 develops the black melanoid pigment typical of the wild type (Heale \& Isaac, 1964). To prevent the development of this pigment the stock cultures were subcultured once a month. M5.6 is one of these subcultures, it possesses the same carotenoids as the original $\mathrm{m} 5$, but in slightly different proportions.

The cultural conditions and the extraction and identification of the carotenoids were as described previously (Valadon \& Heale, 1965). Authentic phytofluene was obtained from Gerbera jamesonii (Valadon, unpublished observation), neurosporene from Neurospora crassa (Haxo, 1949) and $\beta$-carotene and lycopene were provided by Dr O. Isler (Hoffmann-La Roche Ltd., Basle). $\beta$-zeacarotene and $\zeta$-carotene were purified and identified according to the method of Simpson et al. (1963).

Some of the cultures were grown in the presence of $\beta$-ionone or methylheptenone vapours. This was achieved by dipping a cork into these liquids, and touching the cork surface lightly to the inner tip of the cotton wool plugs, which were then re-inserted into the mouths of the conical flasks (Simpson et al. 1964). DPA or 2-hydroxydiphenyl to be incorporated in the medium was dissolved in a few $\mathrm{ml}$. methanol and this solution was then added to the culture medium.

Diphenylamine (DPA) has an absorption maximum in hexane at $282 \mathrm{~m} \mu$; Goodwin (1952) suggested that this interfered with the phytoene fraction in Phycomyces. Turian (1957) treated Neurospora cultures with 1/40,000 DPA+ $\mathrm{Fe}^{2+} \mathbf{0 . 2} \mathrm{mg}$./1. and found that there was no DPA left in the unsaponifiable fraction. Villoutreix (1960) however, found that in his DPA-treated culture of Rhodotorula mucilaginosa, DPA was possibly strongly fixed on to the organisms, and would therefore appear in the phytoene fraction. The phytoene fraction of our cultures of M 5.6 when grown in presence of DPA showed a single maximum absorption peak at $282 \mathrm{~m} \mu$, corresponding to DPA. Phytoene was obtained pure by re-chromatography on $\mathrm{MgO}+$ celite by using purified hexane as developer, collecting $10 \mathrm{ml}$. portions of the eluate and reading these separately.

\section{RESULTS}

The pigments of Verticillium albo-atrum м $\mathbf{5} \cdot \mathbf{6}$ are given in Table $\mathbf{1}$.

\section{Use of inhibitors}

$\beta$-ionone and methylheptenone vapours. Results obtained with these two compounds (Table 1) show much the same pattern, in that the phytoene content was increased considerably while $\gamma$-carotene, lycopene, and neurosporaxanthin were decreased; $\beta$-carotene was the only compound which behaved differently as it increased slightly with $\beta$-ionone and decreased with methylheptenone. The following carotenoids were identified for the first time in mutants of Verticillium: phytofluene, $\beta$-zeacarotene, $\zeta$-carotene, neurosporene. The same amounts of phytofluene and $\zeta$-carotene were obtained in both the treated cultures. Approximately twice as much $\beta$-zeacarotene and neurosporene were obtained in the methylheptenone cultures. 
Table 1. Effect of $\beta$-ionone and methylheptenone vapours on carotenoids of Verticillium albo-atrum $M 5 \cdot 6$ (10-day cultures)

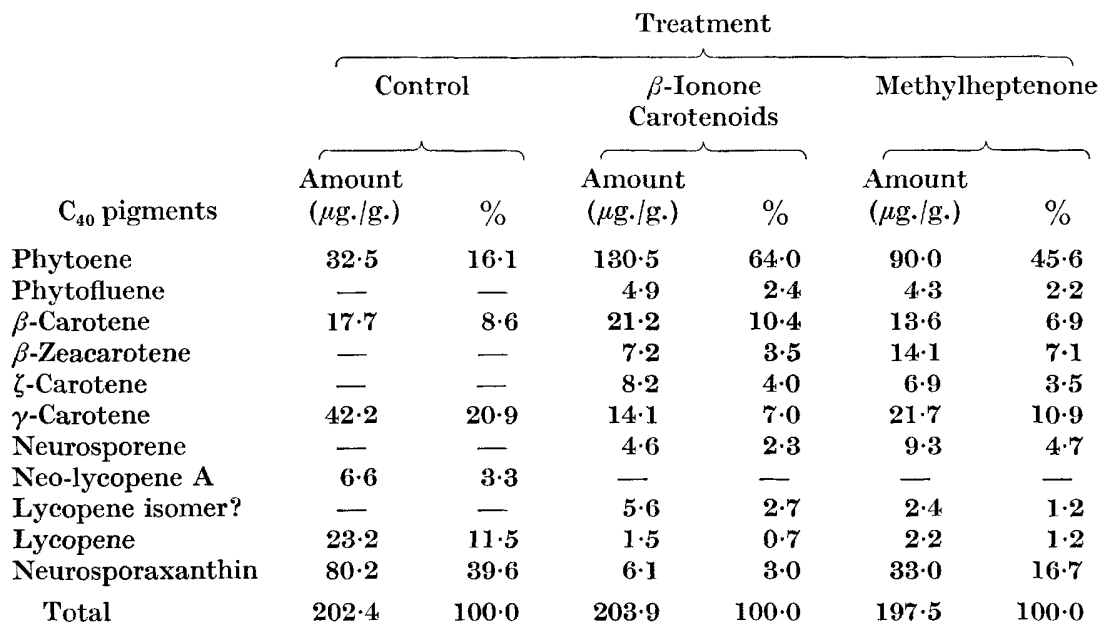

Diphenylamine (DPA). Different concentrations of DPA were used; the results are recorded in Table 2. At a concentration of 1/200,000 DPA, phytoene was increased from 16 to $37 \%$; phytofluene, $\beta$-zeacarotene, $\zeta$-carotene and neurosporene were identified. There was however, a decrease in $\beta$-carotene, $\gamma$-carotene, lycopene and neurosporaxanthin. At the higher concentration of $1 / 25,000 \mathrm{DPA}$, very much the same pattern was obtained: an inhibition of the production of the most unsaturated polyenes ( $\beta$-, $\gamma$-carotene, lycopene, neurosporaxanthin) and a stimulation of the more saturated ones (phytoene, phytofluene, $\beta$-zeacarotene, $\zeta$-carotene, neurosporene).

Table 2. Effect of the concentrations of diphenylamine on carotenoids of Verticillium albo-atrum м5·6 (10-day cultures)

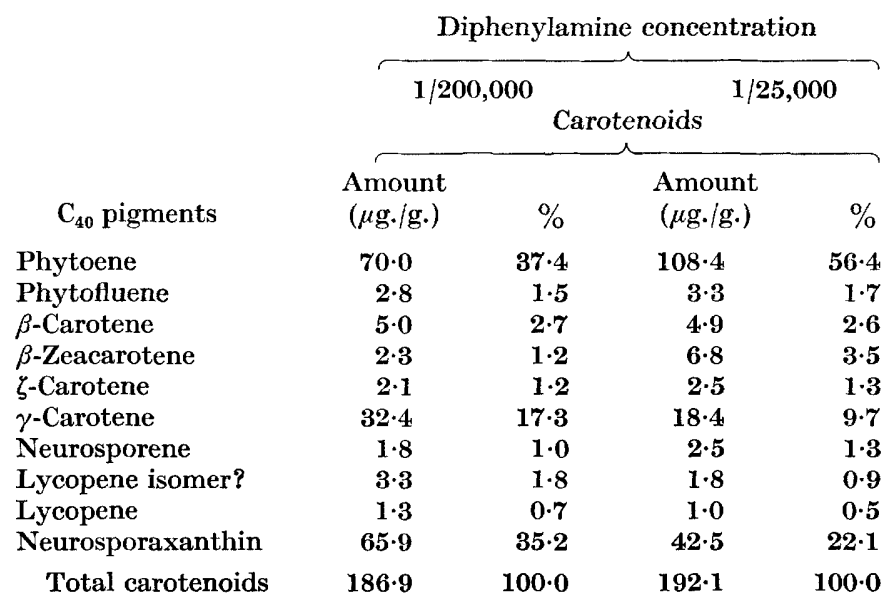




\section{Counteracting the effect of $D P A$}

Slechta, Gabriel \& Hoffmann-Ostenhof (1958) who worked with Rhodotorula gracilis found that inhibition by DPA was annulled when the organisms treated with DPA were transferred to a medium containing a nitrogen source. Goodwin et al. (1953) grew mycelium of Phycomyces blakesleeanus in DPA, washed it thoroughly, incubated it for 1 day in water, transferred to new media, and found that carotenoids appeared. With DPA treated Rhodotorula mucilaginosa cultures, carotenogenesis appeared only after the organisms had grown on a DPA-free medium and were transferred at least five times to new media. Villoutreix (1960) suggested that DPA is strongly fixed to the organisms and when DPA is diluted sufficiently to render it inactive, then carotenoids appear again.

In an attempt to annul the effect of DPA, in the hope of demonstrating the conversion of the more saturated polyenes into the most unsaturated ones, Verticillium albo-atrum м 5.6 was grown in 40 p.p.m. DPA, and after 6 days the cultures were divided into three parts. The first part was analysed for its carotenoid contents (Table 3). The results as compared with strain $\mathbf{m} 5 \cdot 6$ grown in the usual DPA-free medium (Table 3 ) showed the same pattern as before: an inhibition of the formation of the most unsaturated polyenes, a stimulation of the more saturated ones and the appearance of the four carotenes: phytofluene, $\beta$-zeacarotene, $\zeta$-carotene, neurosporene.

The second portion of the culture was washed four times in $\mathrm{M} / \mathbf{1 5}-\mathrm{KH}_{2} \mathrm{PO}_{4}$, transferred to new DPA-free media containing peptone, and the carotenoids estimated after 10 days. The results (Table 3 ) show that the total amounts of carotenoids were much the same, with a high content of the $\mathbf{C}_{40}$ polyene phytoene (slightly higher than after 6 days) and a slight decrease in all the other carotenoids, thus suggesting that the inhibitory effect of DPA has hardly been affected.

Table 3. Effect of diphenylamine (DPA) on Verticillium albo-atrum $M 5 \cdot 6$

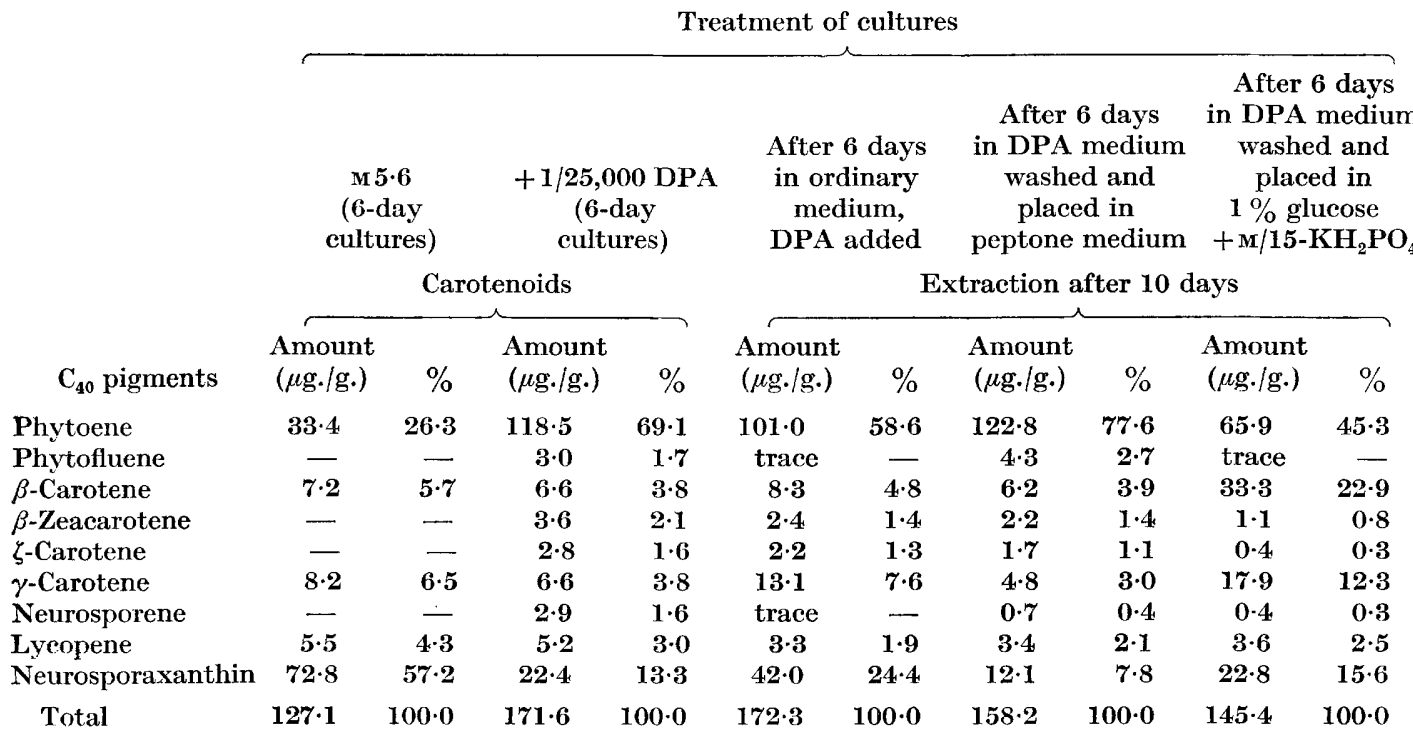


The third portion of the culture was washed in $\mathrm{M} / \mathbf{1 5}$ phosphate buffer 4 times, incubated for one day in water and then transferred to a new medium of $1 \%$ glucose in $\mathrm{M} / \mathbf{1 5}-\mathrm{KH}_{2} \mathrm{PO}_{4}$. These cultures were extracted after 10 days; the results (Table 3 ) show that phytoene and the other saturated polyenes were decreased while $\beta$ carotene, $\gamma$-carotene and neurosporaxanthin were increased.

\section{Action of diphenylamine}

Goodwin et al. (1953) found that adenylic acid and riboflavin annulled the inhibitory effect of DPA in Phycomyces blakesleeanus and suggested that DPA interfered with oxidative phosphorylation in that fungus. Turian (1957) suggested that this interference might only be a secondary effect since other organisms, Mycobacterium phlei (Goodwin, 1953) and Rhodospirillum rubrum (Goodwin \& Osman, 1953) did not show this. With Neurospora crassa, Turian (1957) suggested that as DPA has strong anti-oxidation properties it inhibited the dehydrogenation of precursors of carotenoids. Rilling (1965) suggested that as DPA had the same molecular structure as that of the central part of phytofluene, it might act by binding with the enzyme (which would interact with the more saturated carotenoid to perform the dehydrogenation reaction) and in so doing inhibit the dehydrogenation of the carotenoid. The possibility exists then that if the more unsaturated pigments were already formed, these would not show a decrease.

The following experiment was therefore made.

Verticillium albo-atrum м 5·6 was grown for 6 days in ordinary medium and then 1/25,000 DPA was added. The culture became paler in colour after $12 \mathrm{hr}$. After 4 more days, the carotenoids were extracted; the results given in Table 3 . There was a slight increase in phytoene as compared to that in cultures grown in DPA for 10 days ('Table 2 ), and only a trace of phytofluene and neurosporene. The $\beta$-carotene and lycopene contents were higher but the others were lower except $\zeta$-carotene and neurosporaxanthin which were in more or less the same amounts. When, however, the carotenoid content of cultures grown in DPA-free culture for 6 days and then in DPA is compared with that of strain M $5 \cdot 6$ after 6 days in ordinary DPA-free medium, one notices a high increase in phytoene, a slight increase in $\gamma$-carotene and a decrease in lycopene and neurosporaxanthin.

2-Hydroxydiphenyl. Villoutreix (1960) showed that 2-hydroxydiphenyl was an inhibitor of carotenogenesis in Rhodotorula mucilaginosa, that it did not interfere with growth and that its inhibition could be annulled fairly easily. Rilling (1965), however, did not find any effect of this compound on Mycobacterium phlei. Villoutreix's method was tried with Verticillium albo-atrum $\mathbf{M} 5 \cdot 6$, in an effort to test whether 2-hydroxydiphenyl is inhibitory to Verticillium and if so, what pigments are formed once the inhibition is annulled. The cultures were grown in medium +10 p.p.m. 2-hydroxydiphenyl and after 7 days, one half was used for carotenoid determination (Table 4) while the other half was washed in cold water 4 times, suspended in $\mathrm{M} / \mathbf{1 5}-\mathrm{KH}_{2} \mathrm{PO}_{4}$ solution and incubated for a further 3 days, after which the carotenoids were extracted. The results (Table 4) showed that there was a decrease in phytoene, $\beta$-zeacarotene, $\zeta$-carotene and neurosporene; $\beta$-carotene increased as did $\gamma$-carotene, lycopene and neurosporaxanthin after washing free from 2-hydroxydiphenyl, as compared to 7 -day cultures containing 2-hydroxy- 
diphenyl. 2-Hydroxydiphenyl at higher concentrations namely those used by Villoutreix (1960) of 4-5 $\times 10^{-5}$ inhibited strongly the growth of $V$. albo-atrum м 5.6.

Table 4. Effect of hydroxydiphenyl on carotenoids of Verticillium albo-atrum $\mathrm{M} 5 \cdot 6$

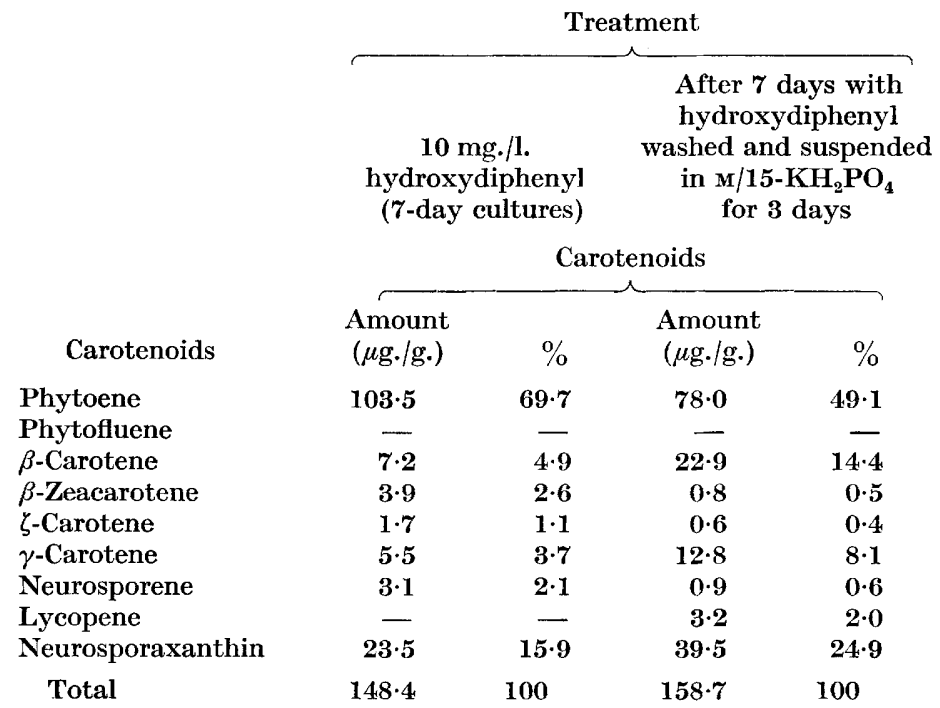

\section{DISCUSSION}

$\beta$-Ionone, a compound which is theoretically capable of providing terminal groups to specific carotenoid molecules, was found to markedly increase $\beta$-carotene production in Phycomyces by Mackinney et al. (1952). Reyes (1963; quoted by Simpson et al. 1964) showed that $\beta$-ionone stimulated carotenoid and sterol formation and suggested that the effect was one of an inhibition of a negative feedback mechanism acting at the pathway level of the phosphorylated derivations of mevalonic acid. An increase in $\beta$-carotene was not obtained by Nakayama et al. (1957) with Syncephalastrum racemosum and Simpson et al. (1964) with Rhodotorula glutinis. The varied effects shown by $\beta$-ionone may be explained on the grounds that it is difficult to control the amount of $\beta$-ionone effectively present in the cultures. At low concentrations $\beta$-ionone appears to stimulate $\beta$-carotene synthesis and at higher concentrations it is inhibitory. In Verticillium albo-atrum $\mathbf{m} \mathbf{5 \cdot 6}$, we found a slight increase in $\beta$-carotene and at the same time a substantial increase in the more saturated compounds and a decrease in the most unsaturated compounds except $\beta$-carotene. Similar results were obtained with methylheptenone. Nakayama et al. (1957) showed in Phycomyces that methylheptenone increased phytoene, phytofluene and $\zeta$-carotene significantly while $\beta$-carotene was only slightly increased. In Syncephalastrum racemosum, however, while the phytoene was increased significantly the $\beta$-carotene decreased appreciably. Simpson et al. (1964) obtained very much the same results as with $\beta$-ionone: stimulation in the formation of phytoene, phytofluene, $\zeta$-carotene, neurosporene and $\beta$-zeacarotene and considerable 
diminution of $\beta$-carotene, torulin and torularhodin. In fact, Simpson et al. seem to be the only workers to have obtained quite such similar results with both compounds. Our results too, with the two compounds, resembled theirs except that there was stimulation of $\beta$-carotene with $\beta$-ionone, and inhibition of it with methylheptenone; associated with this difference in $\beta$-carotene formation, there was much less $\gamma$-carotene (14.1 $\mu \mathrm{g} . / \mathrm{g}$.) with $\beta$-ionone than with methylheptenone (21.7 $\mu \mathrm{g} . / \mathrm{g}$.). Our results would fit in with the hypothesis that the inhibiting compounds block dehydrogenation steps in the Porter-Lincoln pathway, causing the accumulation of the more saturated intermediates. The different amounts of $\beta$-carotene may be due to an additional block between the step $\gamma-\rightarrow \beta$-carotene.

With methylheptenone, $\beta$-ionone and with DPA all the intermediates of the Porter \& Anderson (1962) hypothesis (Fig. 1) from phytoene to $\beta$-carotene, including lycopene and $\beta$-zeacarotene have been isolated with $M 5 \cdot 6$ cultures. The presence of these $\mathrm{C}_{40}$ polyenes does not however prove sequential pathways from phytoene to $\beta$-carotene. With DPA (40 mg./l.) the cultures were still slightly coloured and when washed, incubated in water for one day and transferred to a medium containing $1 \%$ glucose in $\mathrm{M} / \mathbf{1 5}-\mathrm{KH}_{2} \mathrm{PO}_{4}$, the more unsaturated polyenes namely $\beta$-, $\gamma$-carotene and neurosporaxanthin seem to be formed at the expense of the more saturated ones, phytoene, phytofluene, $\zeta$-carotene, neurosporene and $\beta$-zeacarotene. Lycopene, however, is increased from 0.5 to $2.5 \%$, and this might seem to suggest that $\gamma$ carotene is formed via $\beta$-zeacarotene rather than through lycopene.

With hydroxydiphenyl on Rhodotorula mucilaginosa, Villoutreix (1960) showed that most of the more saturated polyenes except phytofluene were identified. When grown on $50 \mu \mathrm{g}$ hydroxydiphenyl/ml., colourless cultures were obtained which still contained phytoene, $\zeta$-carotene and neurosporene. $24 \mathrm{hr}$ after removal of the inhibitor, $\beta$ - and $\gamma$-carotene appeared as well as torulene and torularhodin, but there were still no signs of any phytofluene. The absence of phytofluene made him suggest that the step phytoene $\rightarrow$ phytofluene was doubtful and similarly the step phytofluene $\rightarrow \zeta$-carotene. Our results with the same inhibitor agree very closely with his in that no phytofluene was obtained in cultures treated with hydroxydiphenyl. However, when the effect of the inhibitor was removed there was an increase in $\beta$-, $\gamma$-carotene, lycopene and neurosporaxanthin presumably at the expense of phytoene, $\beta$-zeacarotene, $\zeta$-carotene and neurosporene. With the other inhibitor DPA, we have obtained phytofluene and this tends to suggest that phytofluene may take part in the steps phytoene $\rightarrow$ phytofluene $\rightarrow \zeta$-carotene, though not identified in hydroxydiphenyl inhibited cultures. It is possible that phytofluene was formed and used up rapidly and therefore would not have appeared in the cultures. Our results are, then, consistent with the proposed sequential pathway as suggested by Porter \& Anderson (1962), and also that $\gamma$ - and therefore $\beta$-carotene are synthesized through $\beta$-zeacarotene as suggested by Simpson et al. (1964), Davies et al. (1963) rather than through lycopene.

Our cultures after carotenoid synthesis had started became visibly paler after DPA was added. However, this seems to be due only to loss in amount of neurosporaxanthin by about a half. The more unsaturated pigments that had already been formed do not show a decrease, in fact, a slight increase, and this supports the suggestion made by Turian (1957) and Rilling (1965) that DPA may act in inhibiting the dehydrogenation of precursors of carotenoids. However, it is also well known 
that growth of fungi is affected by DPA and it is very probable that metabolism of carotenoids may not be the only system affected (Villoutreix, 1960), even though Olson \& Knizley (1962) showed that the effect of DPA is apparently restricted to carotenoid biosynthesis, as neither sterols nor fatty acids seem to be affected.

2-hydroxydiphenyl too seems to show different effects on different microorganisms. The growth of Rhodotorula mucilaginosa is not affected at concentrations of $50 \mu \mathrm{g} . / \mathrm{ml}$. (Villoutreix, 1960) while it acts as a good inhibitor of carotenoid synthesis in this organism. Rilling (1965) finds it without any effect on the carotenoids of Mycobacterium phlei and in Verticillium albo-atrum, no growth is observed at $50 \mathrm{mg} . / \mathrm{l}$. while at $10 \mathrm{mg}$./1. it affects carotenoid synthesis.

These examples demonstrating the different effects of the same carotenoid inhibiting compounds on different organisms emphasize the impossibility at least at the moment of obtaining any complete and/or unified picture of carotenoid synthesis in micro-organisms.

We wish to thank Professor C. O. Chichester for the gift of methylheptenone, Dr O. Isler and the firm Hoffman-La Roche, Basle for gifts of $\beta$-carotene and lycopene, and Dr J. E. Kerr for help with the manuscript. A grant from the Central Research Fund of the University of London is gratefully acknowledged.

\section{REFERENCES}

Chichester, C. O., Wong, P. S. \& Mackinney, G. (1954). On the biosynthesis of Carotenoids. Plant Physiol. 29, 238.

Davies, B. H. (1961). The biosynthesis of carotenoids of Rhizophlyctis rosea. Biochem. $J$. 80, 48 P.

Davies, B. H., Villoutreix, J., Williams, R. J. H. \& Goodwin, T. W. (1963). The possible role of $\beta$-zeacarotene in carotenoid synthesis. Biochem. $J .89,96 \mathrm{P}$.

Goodwin, T. W. (1952). Identification of the minor polyene components of the fungus Phycomyces blakesleeanus and a study of their synthesis under various cultural conditions. Biochem. J. 50, 550 .

Goonwin, T. W. (1953). The biogenesis of carotenoids. J. Sci. Fd Agric. 5, 209.

Goodwin, T. W. \& Osman, H. G. (1953). General cultural conditions controlling carotenoid (spirilloxanthin) synthesis in the photosynthetic bacterium Rhodospirillum rubrum. Biochem. J. 53, 541.

Goodwin, T. W., JAmikonN, M. \& Willmer, J. S. (1953). Further observations concerning the action of diphenylamine in inhibiting the synthesis of $\beta$-carotene in Phycomyces blakesleeanus. Biochem. J. 53, 531.

Haxo, F. (1949). Studies on the carotenoid pigments of Neurospora. Arch. Biochem. 20, 400.

Heale, J. B. \& IsAac, I. (1964). Dark pigment formation in Verticillium albo-atrum. Nature, Lond. $202,412$.

Krzeminski, L. F. \& QuaCkEnBush, F. W. (1960). Incorporation of C ${ }^{14}$-labelled compounds into carotenes by Neurospora crassa. Arch. Biochem. Biophys. 88, 287.

Mackinney, G., Nakayama, T., Buss, C. D. \& Chichester, C. O. (1952). Carotenoid production in Phycomyces. J. Am. chem. Soc. 74, 3456.

Nakayama, T., Chichester, C. O., Lukton, A. \& Mackinney, G. (1957). Phytoene production in Phycomyces. Arch. Biochem. Biophys. 66, 310.

Olson, J. A. \& KNIZley, J. H. (1962). The effect of diphenylamine on carotenoid, sterol and fatty acid synthesis in Phycomyces blakesleeanus. Arch. Biochem. Biophys. 97, 138. 
Porter, J. W. \& Anderson, D. G. (1962). The biosynthesis of carotenes. Arch. Biochem. Biophys. 97, 520.

Porter, J. W. \& Lincoln, R. E. (1950). The mechanism of carotene biosynthesis. Arch. Biochem. 27, 390.

Rilling, H. C. (1965). A study of inhibition of carotenoid synthesis. Arch. Biochem. Biophys. 110, 39.

Rüegg, R., Schwieter, U., Ryser, G., Schudel, P. \& Isler, O. (1961). Synthesen in der Carotenoid-Reihe. Helv. chim. Acta 44, 994.

Simpson, K. L., Nakayama, T. O. M. \& Chichester, C. O. (1964). Biosynthesis of yeast carotenoids. J. Bact. 88, 1688.

Slechta, L., Gabriel, O. \& Hoffman-Ostenhof, O. (1958). Separation of carotenoid synthesis from fat synthesis in Rhodotorula gracilis by diphenylamine. Nature, Lond. $181,268$.

Turian, G. (1957). Recherches sur l'action anticaroténogène de la diphénylamine et ses conséquences sur la morphogenèse reproductive chez Allomyces et Neurospora. Physiol. Plant. 10, 667.

Turian, G. \& Haxo, F. (1954). Minor polyene components in the sexual phase of Allomyces javanicus. Bot. Gaz. 115, 254.

Valadon, L. R. G. \& Heale, J. B. (1965). Carotenoids in mutants of Verticillium alboatrum. J. gen. microbiol. 41, 341.

Villoutreix, J. (1960). Sur les Caroténoüdes d'une Levure Rouge: Rhodotorula mucilaginosa (Jorg) Harrison. Nancy: Imprimerie Grandville.

Zalokar, M. (1954). Studies on biosynthesis of carotenoids in Neurospora crassa. Arch. Biochem. Biophys. 50, 71. 